\title{
Effect of electric field on spray deposited CdTe thin films
}

\author{
K. Vamsi Krishna, V .Dutta*, P.D. Paulsonaa, \\ ${ }^{a}$ Photovoltaic Laboratory, Centre for Energy Studies, Indian Institute of Technology Delhi, Hauz Khas, New Delhi 110 016, India \\ bInstitute of Energy Conversion, University of Delaware, Newark, DE 19716, USA
}

Received 16 December 2002; received in revised form 26 May 2003; accepted 13 June 2003

\begin{abstract}
CdTe thin films have been deposited using spray pyrolysis with and without electric field. The improvement in the film properties with the electric field is observed which is mainly due to the reduction of droplet size. The presence of CdTeO peaks in the X-ray diffraction pattern for films deposited without electric field at $3508 \mathrm{C}$ is attributed to the slow dissociation of complexes containing $\mathrm{Cd}$ and $\mathrm{Te}$ ions on the substrate. The reduction in the droplet size under the influence of electric field and faster dissociation of droplets at high temperature leads to complete pyrolytic reaction for a nearly oxide free CdTe film formation. Energy dispersive X-ray analysis indicates stoichiometric $\mathrm{Cd}$ and $\mathrm{Te}$ atomic concentrations, with oxygen and chlorine impurities in varying amount for different substrate temperatures, with and without electric field. The presence of chlorine gives rise to an intense photoluminescence peak at $1.40 \mathrm{eV}$ along with a weak peak at $0.84 \mathrm{eV}$. The intensities of both peaks diminish when the films are prepared with the electric field, due to reduction of chlorine concentration and morphological changes in the films.
\end{abstract}

Keywords: Cadmium telluride; Spray deposition; Electrostatic assisted spray; Solar cells

\section{Introduction}

CdTe thin films are efficient photovoltaic energy converters due to optimum bandgap ( $1.5 \mathrm{eV}$ ) and high solar absorptance wlx. Several thin film deposition techniques have been used for obtaining photovoltaic quality p-CdTe films, which with n-type $\mathrm{CdS}$ window layer have yielded solar cells with conversion efficiency upto $16 \%$ w2x. For large area deposition the techniques requiring vacuum system may not be suited for meeting the goal of a low cost production technology. Chemical spray pyrolysis can be one of the techniques, which may be more suitable for this purpose.

Chemical spray pyrolysis is a low equipment cost technique for depositing thin polycrystalline films of oxides, binary and ternary chalcogenides and superconducting oxide films w3x. The deposition of II-VI semiconductors of sulfides and selenides by spray pyrolysis was first investigated by Chamberlin and Skarman wAx and cadmium telluride films by Boone et al. w5x and Jordan et al. w6x.

The film formation depends on the process of droplet impinging on a heated substrate and the subsequent solvent evaporation leaving the chemical species for film formation on the substrate. The pyrolytic reaction leads to the deposition of a film of the desired compound while all the unreacted species and other reaction products leave the system as volatile components w7x. Depending on the process conditions, heterogeneous andyor homogeneous reaction can take place w\&x. The deposition rate and the thickness of the films can be easily controlled over a wide range by changing the spray parameters like substrate temperature, concentration of the precursor solution and distance between the spray nozzle and the substrate. The properties of the film depend upon the anion to cation ratio, spray rate, substrate temperature, droplet size and also the cooling rate after deposition w3x.

The large droplet size (approx. few tens of microns) in normal spray deposition can result in large splat formation and it causes a slower solvent evaporation. The hydraulic pressure atomisation is capable of producing an average droplet size of $20 \mathrm{~mm} w 9 x$. These 


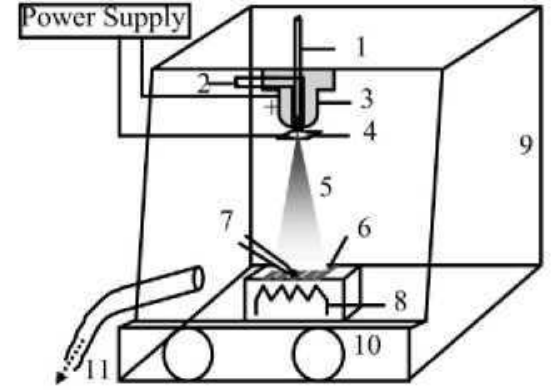

1. Solution inlet 2.Gas inlet 3. Spray Nozzle in Teflon box 4. Metal electrode 5. Spray 6.Substrates 7. Thermocopuple 8.Heating element 9. FRP main body 10. Glove ports 11. Exhaust gases to scrubber

Fig. 1. Schematic diagram of spray deposition chamber.

large droplets can also create voids, inclusions and pin holes in the films, which are detrimental for the photovoltaic device characteristics. One of the methods to overcome such problems is by using nanoparticles precursor, so that the nanoparticles of CdTe combine to form smooth and dense thin films wlox. Another approach can be to apply an electric field between the nozzle and the substrate for creating an additional electric pressure (Electrostatic assisted spray) wllx. The electrostatically charged droplets are propelled towards the grounded substrate. With the help of electric field, finer droplet size $(-5 \mathrm{~mm})$ wl2x and also monodispersed size distribution w13x have been achieved. Since the surface area of the droplet increases, the rate of evaporation also increases and the droplet size can be further reduced during transportation to the substrate. As a result the pyrolitic reaction at the substrate will also be modified and affect the film deposition w14,15x. Since the electric field can be useful in obtaining better spray deposited CdTe films, we have deposited these films with and without electric field. In this article, we report on the role of the electric field during spray deposition of CdTe thin films.

\section{Experimental details}

CdTe films are deposited inside a spray chamber, which is shown in Fig. 1. It is thermally insulating and gas leak-proof chamber made of fiber reinforced plastic (FRP) material. The spray solution and the compressed air are brought into chamber through the connecting tubes into a Teflon box containing the spray nozzle. The spray solution is gravity fed from a bottle kept on the top of the chamber. The flow rate of Nitrogen carrier gas is controlled by throttle valve and measured by high pressure gauge. A $3 \mathrm{~kW}$ heater is employed for heating the glass substrates and the deposition temperature is measured using chromel-alumel thermocouple fixed on a substrate. During the spray, the chamber is sealed and is partially evacuated by means of a rotary pump. The spray effluents before exhausting are circulated through scrubbers containing $\mathrm{NaOH}$ solutions to dissolve $\mathrm{Cd}$ or $\mathrm{Te}$ vapours present in it.

The spray solution is prepared from a mixture of water, ammonium hydroxide, hydrazine hydride and hydrochloric acid. Cadmium and tellurium are added to this mixture in the form of cadmium chloride $\left(\mathrm{CdCl}_{2}\right)$ and tellurium oxide $\left(\mathrm{TeO}_{2}\right)$ with concentration of ; 0.02 M. Hydrazine hydride is used as a reducing agent to obtain Te ions 2wllox Solution $\mathrm{pH}$ value of 11.2 and flow rate of ; $-1-1.5$ mlymin are used for the deposition on cleaned glass substrates as well as $\mathrm{CdS}$ coated glass. The substrate temperature $\left(T_{S}\right)$ is varied from 300 to 400 8C. An electric field is applied by connecting a high voltage power supply between the nozzle and a circular metal electrode placed ; $2 \mathrm{~mm}$ below it. In the following discussion, Film A refers to the film deposited at $3508 \mathrm{C}$ without electric field, whereas Film B refers to the film deposited at the same temperature with electric field $(800 \mathrm{~V}$ between nozzle and the metal electrode). Similarly Films C and D refer to the films deposited at $4008 \mathrm{C}$ with and without the electric field.

Film thickness has been measured using TaylorHobson Talystep instrument. Film thickness ; $2-3 \mathrm{~mm}$ is obtained after $30 \mathrm{~min}$ of spray deposition. X-Ray diffraction patterns are recorded using Glancing angle X-ray diffractometer (Rigaku Giegerplx-Dymax-RBRU200) with monochromatic $\mathrm{Cu} K$ radiation. Optical microscopy images are taken with Clemex vision PE 3.5 optical microscope. Scanning electron micrographs and Energy dispersive X-ray data are taken on Amray 1810 scanning electron microscope attached with Oxford Instruments Energy 200 Energy dipersive spectroscopic analytical system. Midac FT-Photoluminescence Spectrometer system is used for the PL measurements from 0.6 to $1.7 \mathrm{eV}$ at $4.2 \mathrm{~K}$ with $\operatorname{argon}$ ion laser (1 s 514.5 $\mathrm{nm})$ as the excitation source.

\section{Results and discussion}

Thin films of CdTe have been deposited in the presence and absence of electric field at different substrate temperatures by keeping the spray parameters constant. The films grown under applied voltage cover a larger area and have improved deposition efficiency. This is due to the repulsion of electrostatically charged droplets and droplet size reduction due to electrical pressure. The reduction in droplet size is confirmed by the differences in the splats seen in the optical microscopic images for the films deposited with and without electric field at $3508 \mathrm{C}$ for $3 \mathrm{~min}$ (Fig. 2). The liquid droplet on impact with the substrate surface spreads out into a disk shaped structure (or splat) having the reaction material mainly on the rim w7x. The splat diameter depends on the volume and momentum of the droplet, as well as the substrate temperature. A comparison of 

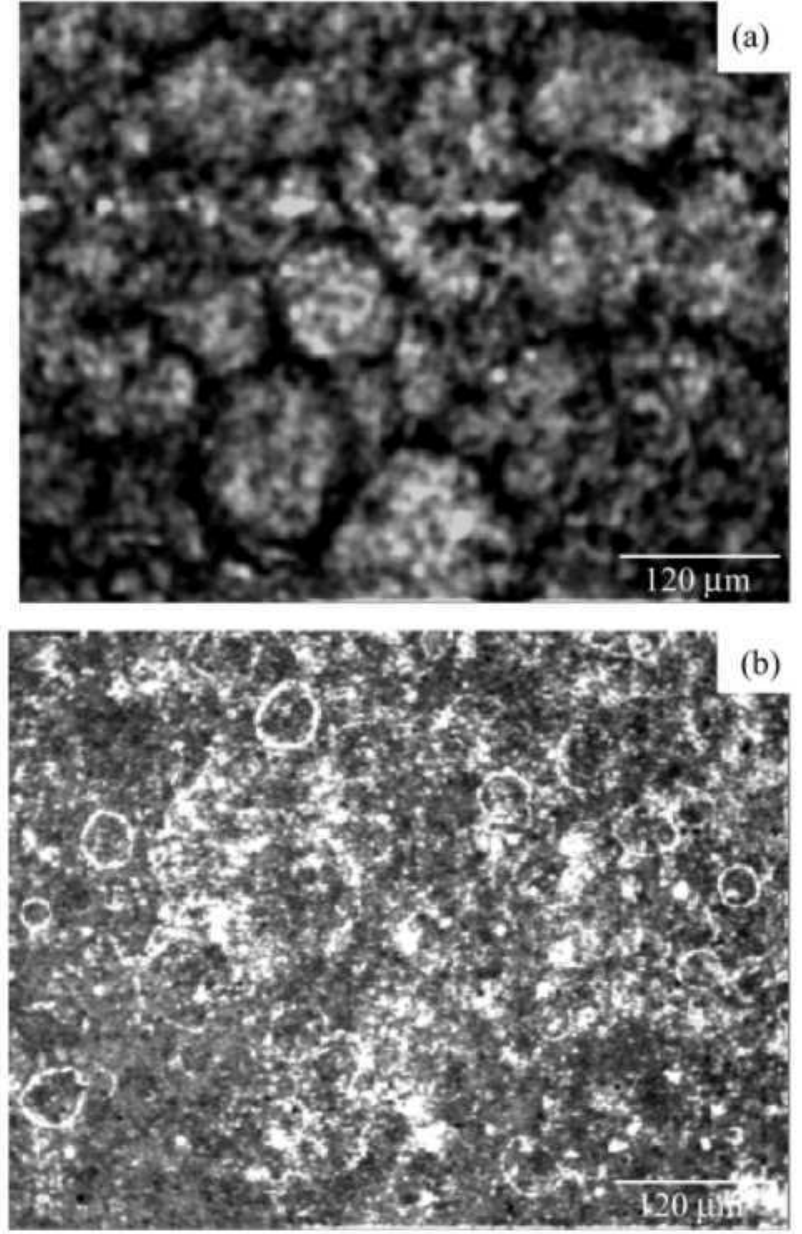

Fig. 2. Optical microscopic images of splat formation on the substrate surface at $\mathrm{T}_{\mathrm{S}} \mathrm{s} 3508 \mathrm{C}$ for films deposited (a) without electric field and (b) with electric field.

Fig. 2a and $\mathrm{b}$ shows that there is a reduction in the splat diameter, which means that the droplet size is indeed reduced under the electric field.

Scanning electron micrographs of films deposited with and without electric field at $T_{S} s 3508 \mathrm{C}$ are shown in Fig. $3 a$ and $b$. The films display distinct surface morphologies, which arise due to the variation in the droplet size. The films grown under an electric field has well formed grains compared to the film grown without the field. This may be due to the fact that since under the electric field there is a reduction in droplet size, after striking the heated substrate the solvent quickly evaporates completing the pyrolytic chemical reaction. Since this difference in film growth process due to droplet size difference happens throughout the film deposition, there will be difference in the microstructure of the films. In fact, cross sectional SEM micrographs (Fig. 4) show large voids in films deposited without field compared to the film with field. The CdS film columnar structure is also seen at the bottom of micrographs.
Fig. 5a and b show the X-ray diffractogram for the films deposited with and without electric field at $T_{S} s$ $3508 \mathrm{C}$, respectively. In both the films CdTe deposits with Zinc blende (Cubic) structure lattice constant of $6.483 \mathrm{~A}$ is nearly equal to the powder value of $6.481 \mathrm{~A}$ w17x. The (111), (220) and (311) are the prominent peaks for film A, which means that there is no preferred orientation. As can be seen in Fig. 5a additional peaks having considerable intensity are present, which correspond to $\mathrm{CdTeO}$. Since the spray deposition has been done under nitrogen environment, there is little possibility of oxygen getting incorporated in the films from the ambient. One possible way by which an oxide phase can be formed is during the film growth. $\mathrm{Cd}$ and $\mathrm{Te}$ ions in the spray solution form complexes such as $\mathrm{Cd}(\mathrm{OH})_{2} 23, \mathrm{TeO}$ and $\mathrm{TeO} 2 \mathrm{q}$ w18x. If these complexes dissociate slowly, it can lead to the formation of secondary $\mathrm{CdTeO}$ phase along with CdTe. This slow dissociation will happen if the substrate temperature is low and the droplet size is large as observed for film A. However, it can be seen from Fig. 5b that the peaks related to
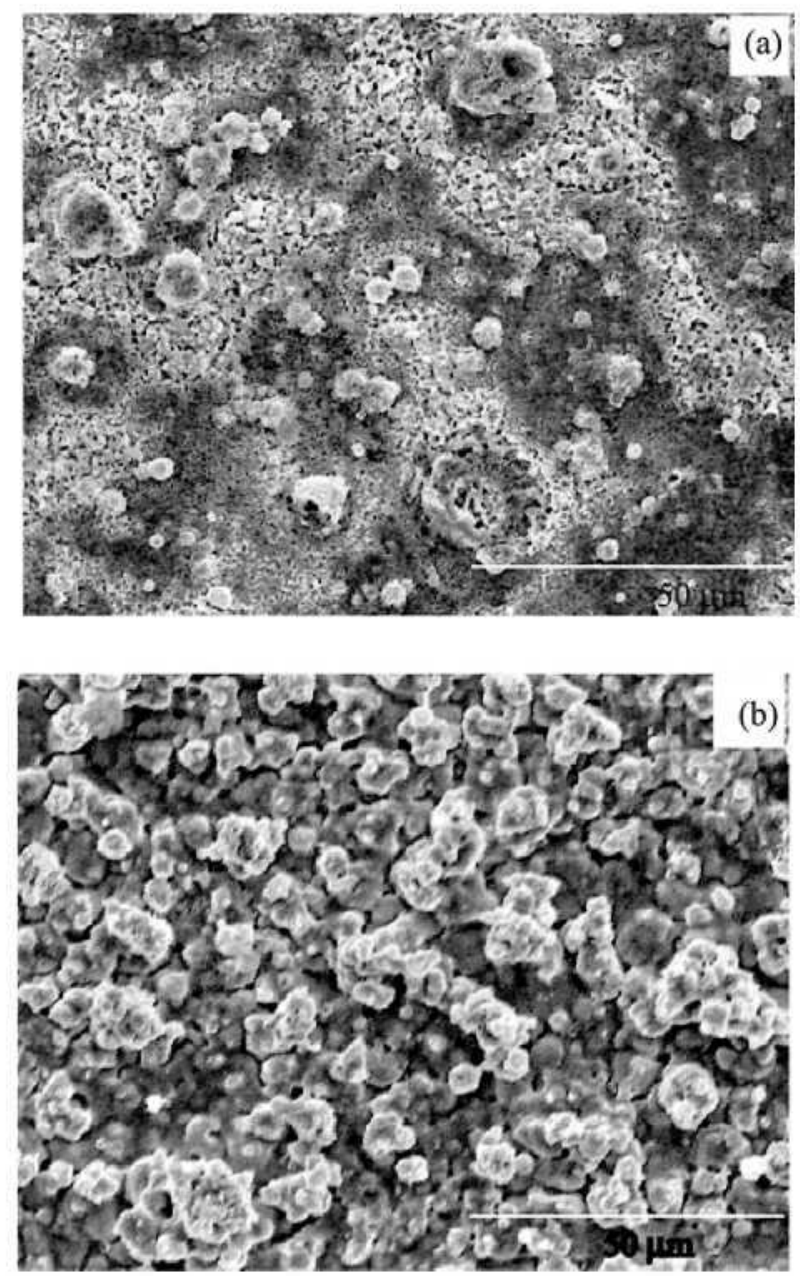

Fig. 3. SEM micrographs of films deposited at $T_{S} s 3508 \mathrm{C}$ (a) without electric field and (b) with electric field. 

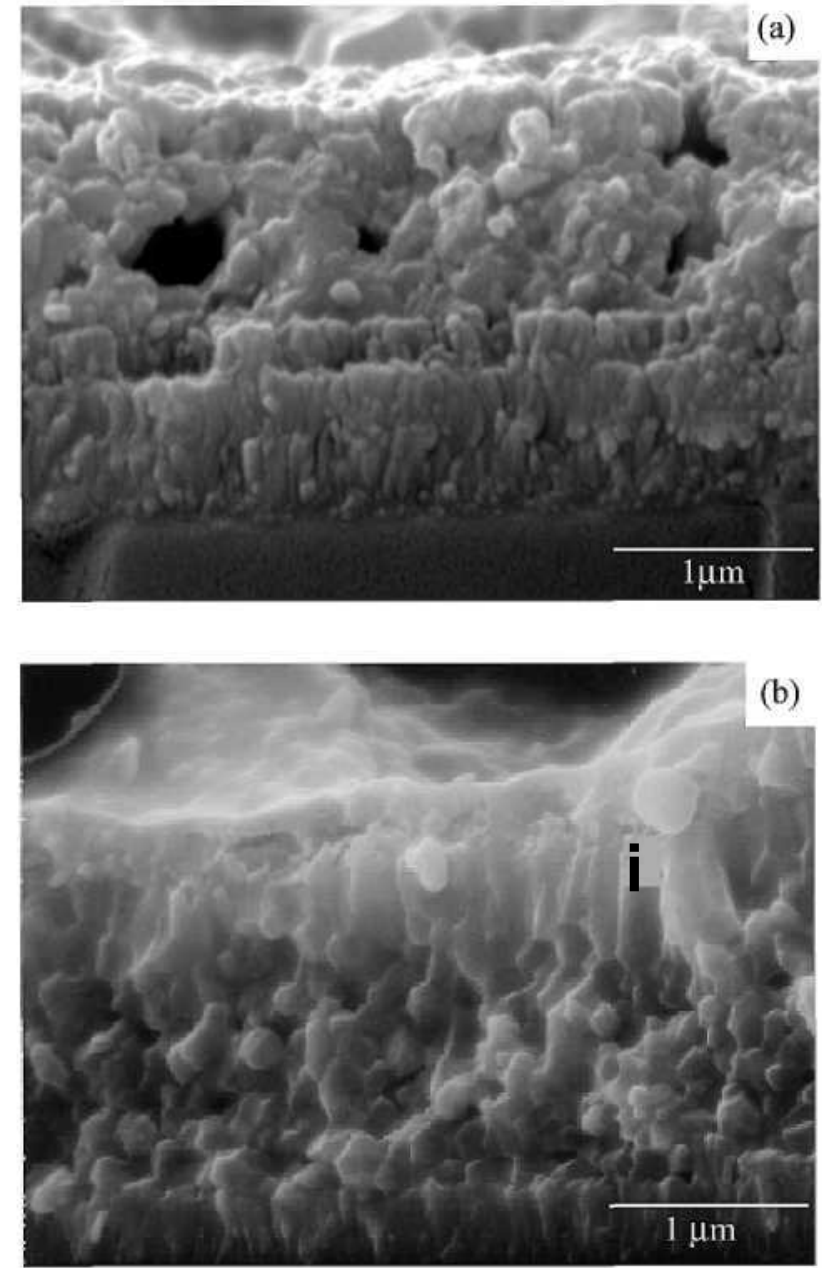

Fig. 4. Cross sectional SEM (Scanning Electron Microscopy) micrographs of films deposited at $\mathrm{T}_{\mathrm{S}} \mathrm{s} 3508 \mathrm{C}$ (a) without electric field and (b) with electric field.

CdTe are unchanged in the presence of electric field. But there is a drastic reduction in $\mathrm{CdTeO}$ peak intensity. It should be noted that the droplet size is small to begin with due to the presence of electric field and can be further reduced while transporting to the substrate. Thus, on arrival at the substrate the complexes will dissociate fast, leaving $\mathrm{Cd}$ and $\mathrm{Te}$ ions, which combine to form nearly oxide free CdTe film.

In order to confirm the role of droplet size and evaporation rate in oxide phase formation, the films are deposited at $\mathrm{T}_{\mathrm{S}} \mathrm{s} 4008 \mathrm{C}$ without (film C) and with the electric field (film D), the corresponding X-ray diffractogram are presented in Fig. 6a and b, respectively. For film $\mathrm{C}$ there is a reduction in oxide peaks as compared to the peaks in films deposited at a substrate temperature of $3508 \mathrm{C}$. In addition, the intensity of (111) peak is higher at $4008 \mathrm{C}$ that suggests an oriented growth. The reduction in oxide peak intensities confirms that a faster solvent evaporation occurring at higher temperatures results in inhibition of $\mathrm{CdTeO}$ formation. Reduced

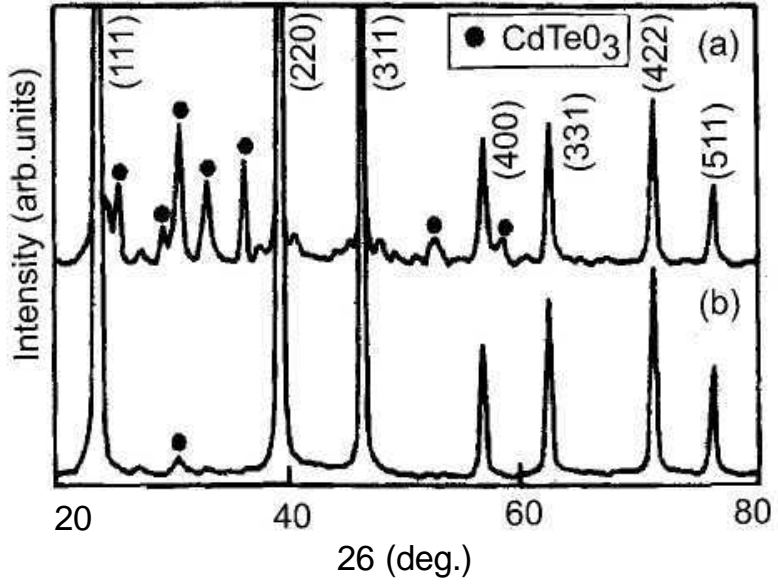

Fig. 5. X-ray Diffraction patterns of films deposited at $T_{S} s 3508 \mathrm{C}$ (a) without electric field and (b) with electric field.

droplet size under electric field adds to this effect reducing $\mathrm{CdTeO}$ intensities further, as can be seen in Fig. 6b. It should be noted that when the droplet size is reduced (film D) under electric field, the X-ray diffractogram becomes similar to that for film B. This means that though the fast solvent evaporation at higher temperatures does reduce possibility of oxide formation, the droplet size reduction has more significant effect. This is further supported by the X-ray diffraction pattern for the films deposited at $\mathrm{T}_{\mathrm{S}} \mathrm{s} 3008 \mathrm{C}$ (Fig. 7), which also shows the intense oxide peak without the electric field and its reduction under the electric field. CdTe films have also been deposited with oxygen as a carrier gas to verify if the oxide formation can take place at 400 $8 \mathrm{C}$ without the electric field. Again this also leads to the formation of polycrystalline CdTe with only small oxide peaks in X-ray diffractogram. This confirms that the formation of $\mathrm{CdTeO}$ does not occur due to the

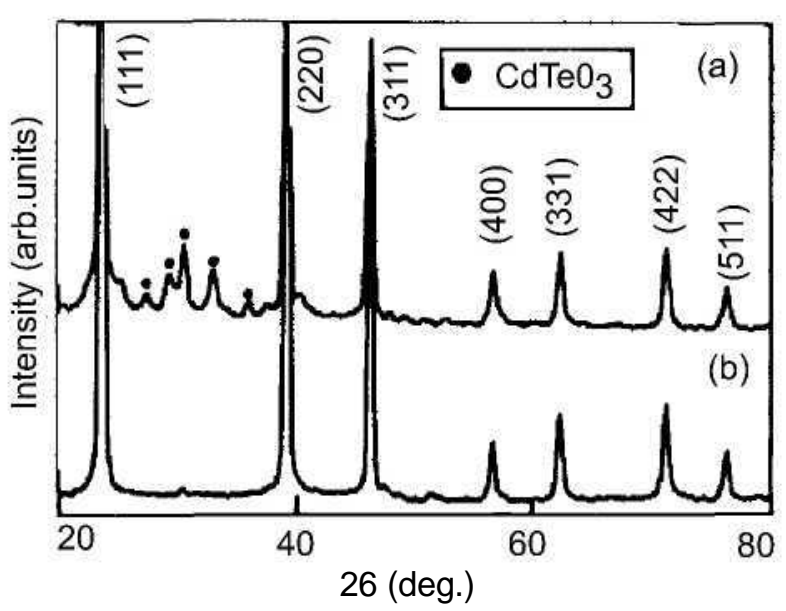

Fig. 6. X-ray Diffraction patterns of films deposited at $T S_{s}=4008 \mathrm{C}$ (a) without electric field and (b) with electric field. 


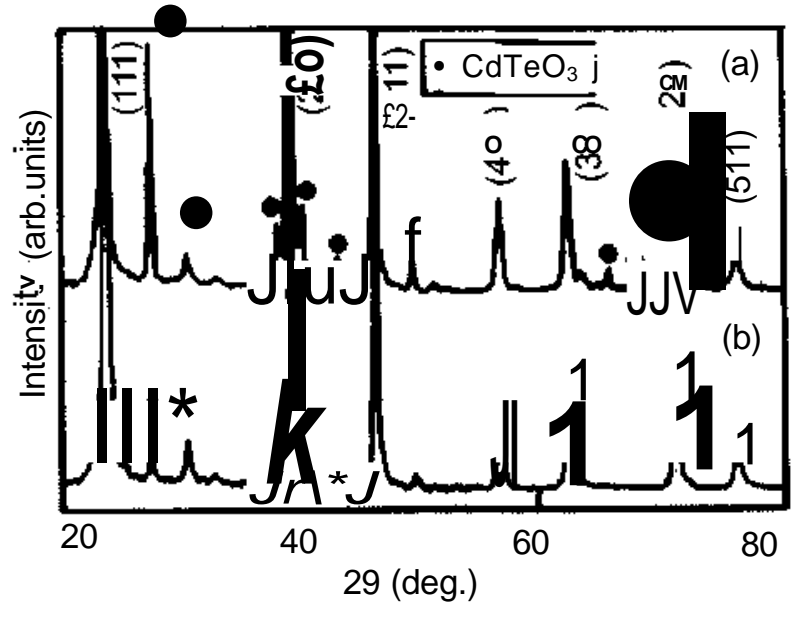

Fig. 7. X-ray Diffraction patterns of films deposited at $T_{S} s 3008 C$ (a) without electric field and (b) with electric field.

reaction with oxygen from the ambient, but due to chemical processes occurring at the substrate itself.

The chemical composition of these films is shown in Table 1. In addition to $\mathrm{Cd}$ and $\mathrm{Te}, \mathrm{O}$ and $\mathrm{Cl}$ are present in the films in significant amounts. A drastic difference is evident for $\mathrm{O}$ and $\mathrm{Cl}$ atomic concentrations in films deposited with and without electric field. In confirmation with X-ray diffraction, the amount (at.\%) of oxygen $30.0 \%$ and chlorine $6.9 \%$ in case of film deposited without electric field at $\mathrm{T}_{\mathrm{S}} \mathrm{s} 3508 \mathrm{C}$ is reduced to $9.4 \%$ and $1.7 \%$, respectively, for film deposited with electric field. The amount of oxygen is $14.4 \%$ for the case of film deposited without electric field at $\mathrm{T}_{\mathrm{S}} \mathrm{s} 400$ 8C, which is reduced to $6.9 \%$ for film deposited with electric field, but the variation in chlorine concentration is not much.

Chlorine is a donor impurity for CdTe material and also it forms complexes with cation vacancies, which should lead to the formation of impurity levels in the bandgap. These levels can be detected using photoluminescence (PL). The PL spectra of films deposited without (film A) and with electric field (film B) at $\mathrm{T}_{\mathrm{S}} \mathrm{s} 3508 \mathrm{C}$ are shown in the Fig. 8a and b, respectively. No PL peak from band to band transition at $1.59 \mathrm{eV}$ for CdTe is observed in both the cases. The most intense emission for film A occurs at $1.40 \mathrm{eV}$, which has also been observed in CdTe thin films prepared by other methods. This peak is much reduced in case of film B and is also shifted to $1.41 \mathrm{eV}$. Another satellite peak at $0.84 \mathrm{eV}$ is observed in film A, which is completely absent in film B.

The $1.40 \mathrm{eV}$ luminescence in CdTe has been associated with atomic complexes involving Te vacancies, $\mathrm{Cd}$ vacancies or alternatively $\mathrm{Cd}$ interstitials. The complex $\left(\mathrm{V}_{\mathrm{cd}}-\mathrm{X}_{\mathrm{Te}}\right)$ formation has been found to give this peak, where $\mathrm{X}$ is a donor atom such as $\mathrm{Cl}$ w19x. The variation in the intensity of $1.41 \mathrm{eV}$ emission with $\mathrm{Cl}$ concentra-
Table 1

Elemental composition of films deposited at $T_{S} s 3508 \mathrm{C}$ (A) without electric field, (B) with electric field and films deposited at $\mathrm{T}_{\mathrm{S}} \mathrm{s} 400$ 8C (C) without electric field, (D) with electric field

\begin{tabular}{lllcl}
\hline $\begin{array}{l}\text { Film } \\
\text { name }\end{array}$ & $\begin{array}{l}\text { Cd } \\
\text { (at.\%) }\end{array}$ & $\begin{array}{l}\text { Te } \\
\text { (at.\%) }\end{array}$ & $\begin{array}{l}\text { O } \\
\text { (at.\%) }\end{array}$ & $\begin{array}{l}\text { Cl } \\
\text { (at.\%) }\end{array}$ \\
\hline A & 30.6 & 30.8 & 30.0 & 6.9 \\
B & 44.2 & 42.6 & 9.4 & 1.7 \\
C & 41.1 & 40.2 & 14.4 & 1.5 \\
D & 45.9 & 43.4 & 6.9 & 2.2 \\
\hline
\end{tabular}

tion is reported by Popovich et al. w20x. Feldman et al. w21x reported $1.41 \mathrm{eV}$ peak due to the defect sites located in intergranular regions in CdTe thin films deposited at $4258 \mathrm{C}$ by spray pyrolysis. Since there is reduction in $\mathrm{Cl}$ impurity in film $\mathrm{B}$ compared to film $\mathrm{A}$, as well as there is an improvement in morphology, the $1.40 \mathrm{eV}$ emission is suppressed and the peak intensity is much reduced. The $0.84 \mathrm{eV}$ deep level is reported by Krustok et al. w22x in $\mathrm{Cu}$ and $\mathrm{Cl}$ doped CdTe powder. We also observed the same peak in film A, which can be attributed to the presence of $\mathrm{Cl}$ in the film. But in film $\mathrm{B}$ the peak is absent due to the reduction of $\mathrm{Cl}$ content in the film.

\section{Conclusions}

Improvement in the structural, compositional, surface morphological and luminescence properties of CdTe thin film deposited by spray pyrolysis under the electric field is observed. The absence of oxide phase in the film deposited under electric field clearly shows that chemical reaction at the substrate is influenced by the reduction in the droplet size. The influence of electric field is more than that due to increased substrate temperature.

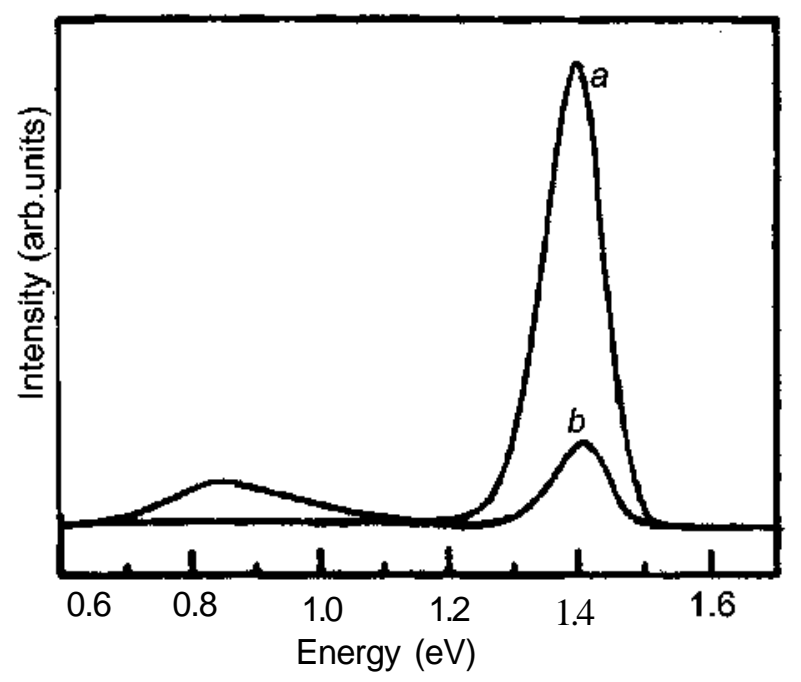

Fig. 8. Photoluminescence spectra of films deposited at $\mathrm{T}_{\mathrm{S}} \mathrm{s} 3508 \mathrm{C}$ (a) without electric field and (b) with electric field. 
Surface morphology and microstructure of the films can be controlled by applying electric field in addition to other spray parameters. In fact, it is possible to achieve an oxide free film at a temperature as low as $3008 \mathrm{C}$ by applying sufficiently high electric field to produce droplets of appropriate size. In ideal spray deposition the droplet should vaporize before striking the substrate and then the material formed should get deposited. Suitable spray parameters and electric field value need to be established to achieve this. Photovoltaic quality CdTe thin films at low temperature deposition under the electric field will lead to the reduction of thermal budget and make it possible to deposit on low cost substrates.

\section{Acknowledgments}

The work reported has been initiated under a project supported by Ministry of Non-Conventional Energy Sources (MNES), New Delhi. One of the authors (K. Vamsi Krishna) wishes to acknowledge MNES, New Delhi for providing the financial assistantship under the National Renewable Energy Fellowship programme. We also wish to acknowledge The Abdus Salam International Centre for Theoretical Physics (ICTP), Trieste, Italy for sponsoring our visit under associate and young collaborator programme, during which the data analysis was done. The authors are grateful to Dr K.S.R. Koteswara Rao of IISc, Bangalore for photoluminescence measurements and Maggie Paulose for SEM measurements.

\section{References}

wx L.J. Joseph, J. Appl. Phys. 27 (1956) 777.

w2x J. Britt, C. Ferekides, Appl. Phys. Lett. 62 (1993) 2851.

w3x P.S. Patil, Mater. Chem. Phys. 59 (1999) 185.

WAx R. Chamberlin, H. Skarman, J. Electrochem. Soc. 113 (1966) 86.

wx J.L. Boone, T.P Vandoren, A.K. Berry, Thin Solid Films 87 (1982) 259.

w6x J.F. Jordan, S.P Albright, Sol. Cells 23 (1988) 107.

wx K.L. Chopra, R.C. Kainthla, D.K. Pandya, A.P Thakoor, Phys. Thin Films 12 (1982) 167.

u\&k J.C. Viguie, J. Spitz, J. Electrochem. Soc. 122 (1975) 585.

w\% G. Blandenet, M. Court, Y. Lagarde, Thin Solid Films 77 (1981) 81.

wl0x M. Pehnt, D.L. Schulz, C.J. Curtis, K.M. Jones, D.S. Ginley, Appl. Phys. Lett. 67 (1995) 2176.

wllx A.H. Lefebvre, Atomization and Sprays, Hemisphere publishing corporation, New York, 1989.

wl2x W. Siefert, Thin Solid Films 120 (1984) 267.

w13x K. Tang, A. Gomez, Phys. Fluids 6 (1994) 2317.

w14x W. Siefert, Thin Solid Films 120 (1984) 275.

w15x D. Zaouk, Y Zaaar, A. Khoury, C. Linares, J.P Charles, J. Bechara, J. Appl. Phys. 87 (2000) 7359.

wl6x S.M. Kulify, J. Amer. Chem. Soc. 83 (1961) 4916.

w17x K. Zanio, in: R.K. Willardson, A.C. Beer (Eds.), Semiconductors and Semimetals, Cadmium Telluride, 13, Academic Press, New York, 1978.

wl8x K. Murase, T. Honda, M. Yamamoto, T. Hirato, Y Awakura, J. Electrochem. Soc. 148 (2000) C203-C210.

w19x D.P Halliday J.M. Eggleston, K. Durose, J. Cryst. Growth 186 (1998) 543

[20] YD. Popovich, G.M. Grigorovich, P.M. Peleshchak, P.N. Tkachuk, Semiconductors 36 (2002) 636.

w21x B.J. Feldman, J.L. Boone, T. Van Doren, Appl. Phys. Lett. 38 (1981) 704.

w22x J. Krustok, A. Loo, T. Piibe, J. Phys. Chem. Solids 52 (1991) 1037. 